Appl. Set-Valued Anal. Optim. 1 (2019), No. 1, pp. 39-52

Available online at http://asvao.biemdas.com

https://doi.org/10.23952/asvao.1.2019.1.04

\title{
STRONG CONVERGENCE THEOREMS FOR $\delta$-INVERSE STRONGLY ACCRETIVE OPERATORS IN BANACH SPACES
}

\author{
SHAHRAM REZAPOUR*, SEYYED HASAN ZAKERI \\ Department of Mathematics, Azarbaijan Shahid Madani University, Tabriz, Iran
}

\begin{abstract}
By using an iteration method, we study some variational inequalities including an $\delta$-inverse strongly accretive operator in Banach spaces. We present a strong convergence theorem for the production sequence method.

Keywords. $\delta$-inverse strongly accretive operator, Monotone mapping, Strong convergence, Variational inequality problems.
\end{abstract}

2010 Mathematics Subject Classification. 47H05, 47J20.

\section{INTRODUCTION}

Let $D$ be a nonempty subset of a real Hilbert space $H$ and $P_{D}$ the metric projection of $H$ onto $D$. A mapping $B: D \rightarrow H$ is said to be monotone if

$$
\langle B u-B v, u-v\rangle \geq 0, \quad \forall u, v \in D .
$$

A mapping $B: D \rightarrow H$ is said to be $L$-Lipschitz if there exits $L \geq 0$ such that

$$
\|B u-B v\| \leq L\|u-v\|, \quad \forall u, v \in D .
$$

If $L=1$ then $B$ is called a nonexpansive mapping. We denote by $F(B)$ the set of fixed points of $B . B$ is said to be $\delta$-inverse strongly monotone, if there exists a positive real number $\delta$ such that

$$
\langle B u-B v, u-v\rangle \geq \delta\|B u-B v\|^{2}, \quad \forall u, v \in D .
$$

It is obvious that any $\delta$-inverse strongly monotone $B$ is Lipschitz and $\|B u-B v\| \leq \frac{1}{\delta}\|u-v\|$. Let $D$ be a nonempty closed convex subset of $H$ and let $B: D \rightarrow H$ be a nonlinear mapping. The variational inequality problem is to find a point $\tilde{x} \in D$ such that

$$
\langle x-\tilde{x}, B \tilde{x}\rangle \geq 0, \quad \forall x \in D .
$$

We denote the solution set of variational inequality problem by $\operatorname{VI}(D, B)$. We note that, if $D=H$ then $V I(H, B)=B^{-1}(0)$, where $B^{-1}(0)=\{x \in H: B x=0\}$. Let $I$ be identity mapping and $T$ be a nonexpansive mapping of $D$ into itself. If $B=I-T$, then $B$ is $\frac{1}{2}$-inverse strongly monotone and $F(T)=V I(D, B)$; see [10]. A mapping $T: D \rightarrow D$ is said to be strictly pseudocontractive if there exists $0 \leq k<1$ such that

$$
\|T u-T v\|^{2} \leq\|u-v\|^{2}+k\|(I-T) u-(I-T) v\|^{2}, \quad \forall u, v \in D .
$$

\footnotetext{
* Corresponding author.
}

E-mail address: rezapourshahram@yahoo.ca (S. Rezapour).

Received February 8, 2019; Accepted March 13, 2019. 
Let $T: D \rightarrow D$ be a strictly pseudocontractive. If $B=I-T$, then $B$ is $\frac{1-k}{2}$-inverse strongly monoton and $F(T)=V I(D, B)$; see [20]. Since Stampacchia introduced variational inequality 1.1 in 1964, many authors extensively studied it from viewpoint of existence, stability, solution methods etc; see, e.g., $[1,2,5,6,7,8,9,11,12,13,16,17,19,20]$ and the references therein. In 2004, Iiduka, Takahashi and Toyoda [10] introduced the following iterative algorithm

$$
x_{1} \in D, \quad x_{n+1}=P_{D}\left(\lambda_{n} x_{n}+\left(1-\lambda_{n}\right) P_{D}\left(x_{n}-\alpha_{n} B x_{n}\right)\right), \quad \forall n \geq 1,
$$

$P_{D}$ is a the metric projection from $H$ onto $D$ and $B: D \rightarrow H$ is $\delta$-inverse strongly monotone, $\lambda_{n} \in[a, b]$ for some $a, b \in(-1,1)$ and $\alpha_{n} \in[c, d]$ for some $c, d \in(0,2(1+a) \delta)$. They proved that the sequence $\left\{x_{n}\right\}$ generated by (1.2) converges weakly to some element of $V I(D, B)$.

In 2005, Iiduka and Takahashi [11] introduced the following iterative algorithm for finding solutions to a variational inequality involving a $\delta$-inverse strongly monotone mapping and obtained the strongly convergence of the following iterative process

$$
x_{1} \in D, \quad x_{n+1}=\lambda_{n} x+\left(1-\lambda_{n}\right) P_{D}\left(x_{n}-\alpha_{n} B x_{n}\right), \quad \forall n \geq 1,
$$

where $B: D \rightarrow H$ is a $\delta$-inverse strongly monotone mapping, $\lambda_{n} \in[0,1)$ with $\lim _{n \rightarrow \infty} \lambda_{n}=0, \sum_{n=1}^{\infty} \lambda_{n}=\infty$ and $\sum_{n=1}^{\infty}\left|\lambda_{n+1}-\lambda_{n}\right|<\infty$ and $\alpha_{n} \in[c, d]$ for some $c, d \in(0,2 \delta)$ with $\sum_{n=1}^{\infty}\left|\alpha_{n+1}-\alpha_{n}\right|<\infty$. They proved that the sequence $\left\{x_{n}\right\}$ generated by (1.3) converges strongly to $P_{V I(D, B)} x$. Recently, Alghamdi, Shahzad and Zegeye [1] suggested the following iterative algorithm for finding a common solution of two variational inequality problems involving Lipschitz monotone mappings $B_{1}, B_{2}$

$$
\left\{\begin{array}{l}
x_{1} \in D \\
z_{n}=P_{D}\left(x_{n}-\alpha_{n} B_{2} x_{n}\right), \\
y_{n}=P_{D}\left(x_{n}-\alpha_{n} B_{1} x_{n}\right), \\
x_{n+1}=\lambda_{n} f\left(x_{n}\right)+\left(1-\lambda_{n}\right)\left(a_{n} x_{n}+b_{n} P_{D}\left(x_{n}-\alpha_{n} B_{1} y_{n}\right)+c_{n} P_{D}\left(x_{n}-\alpha_{n} B_{2} z_{n}\right)\right), \quad \forall n \geq 1,
\end{array}\right.
$$

where $f: D \rightarrow D$ is a contraction mapping, $\alpha_{n} \subset[a, b] \subset\left(0, \frac{1}{L}\right)$ for $L:=\max \left\{L_{1}, L_{2}\right\}\left(L_{1}, L_{2}\right.$ Lipschitz constants). They proved that the sequence $\left\{x_{n}\right\}$ generated by (1.4) converge to a point $q \in V I\left(D, B_{1}\right) \cap$ $V I\left(D, B_{2}\right)$.

Next, we turn our attention to the following generalized variational inequality problem in the framework of Banach spaces. Let $X$ be a Banach space and let $X^{*}$ the dual space of $X$. Let $D$ be a nonempty closed convex subset of $X$. The generalized variational inequality problem is to find a point $\tilde{x} \in$ $D, j(x-\tilde{x}) \in J(x-\tilde{x})$ such that

$$
\langle j(x-\tilde{x}), B \tilde{x}\rangle \geq 0, \quad \forall x \in D .
$$

We use $S(D, B)$ to denote the solution set of this problem, that is

$$
S(D, B)=\{\tilde{x} \in D:\langle j(x-\tilde{x}), B \tilde{x}\rangle \geq 0, x \in D\} .
$$

In 2006, Aoyama, Iiduka and Takahashi [2] investigated the following iterative algorithm

$$
x_{1}=x \in D, \quad x_{n+1}=\lambda_{n} x_{n}+\left(1-\lambda_{n}\right) Q_{D}\left(x_{n}-\alpha_{n} B x_{n}\right), \quad \forall n \geq 1,
$$

where $D$ is a nonempty closed convex subset of a uniformly covex and 2-uniformly smooth Banach space $X$ and $B: D \rightarrow X$ is an $\delta$-inverse strongly accretive operator, $Q_{D}$ is a sunny nonexpansive retraction 
from $X$ onto $D, \lambda_{n} \in[a, b]$ for some $a, b \in(0,1)$ and $\alpha_{n} \in\left[c, \frac{\delta}{k^{2}}\right]$ for some $c>0$. They showed that if $S(D, B) \neq \emptyset$, then the sequence $\left\{x_{n}\right\}$ generated by (1.6) converges weakly to some element of $S(D, B)$.

In 2011, Yao et al. [24] obtained a strong theorem in a uniformly convex and 2-uniformly smooth Banach space. They suggested the following iterative algorithm

$$
x_{1} \in D, \quad x_{n+1}=\lambda_{n} x_{n}+\left(1-\lambda_{n}\right) Q_{D}\left[\gamma_{n} v+\left(1-\gamma_{n}\right) Q_{D}\left(x_{n}-\alpha_{n} B x_{n}\right)\right], \quad \forall n \geq 0,
$$

where $D$ is a nonempty closed convex subset of uniformly covex and 2-uniformly smooth Banach space $X$ and $B: D \rightarrow X$ is an $\delta$-inverse strongly accretive operator with $S(D, B) \neq \emptyset, Q_{D}$ is a sunny nonexpansive retraction from $X$ onto $D, \lambda_{n} \in[a, b]$ for some $a, b \in(0,1)$ with $\lim _{n \rightarrow \infty} \lambda_{n}=0, \sum_{n=1}^{\infty} \lambda_{n}=\infty$ and $\sum_{n=1}^{\infty}\left|\lambda_{n+1}-\lambda_{n}\right|<\infty$ and $\alpha_{n} \in\left[c, \frac{\delta}{k^{2}}\right]$ for some $c>0$ with $\sum_{n=1}^{\infty}\left|\alpha_{n+1}-\alpha_{n}\right|<\infty, v \in X$ is fixed. They proved that the sequence $\left\{x_{n}\right\}$ generated by (1.7) converges strongly to $\tilde{Q}_{D} v$, where $\tilde{Q}_{D}$ is a sunny nonexpansive retraction of $X$ onto $S(D, B)$.

Assume that $B$ is an $\delta$-inverse strongly accretive operator from $D$ a nonempty, closed and convex subset of a uniformly convex and 2-uniformly smooth Banach space $X$, into $X$ with $S(D, B) \neq \emptyset$ and $Q_{D}$ is a sunny nonexpansive retraction from $X$ onto $D$. In this paper, for solving the generalized variational inequality problem involving an $\delta$-inverse strongly accretive operator, we introduce the following iterative algorithm in Banach spaces

$$
\left\{\begin{array}{l}
x_{1} \in D \\
z_{n}=Q_{D}\left(x_{n}-\alpha_{n} B x_{n}\right) \\
\tilde{x}_{n}=\lambda_{n} x_{n}+\left(1-\lambda_{n}\right) Q_{D}\left[\gamma_{n} z_{n}+\left(1-\gamma_{n}\right) Q_{D}\left(z_{n}-\alpha_{n} B z_{n}\right)\right] \\
x_{n+1}=\eta_{n} v+\left(1-\eta_{n}\right)\left(a_{n} x_{n}+b_{n} z_{n}+c_{n} \tilde{x}_{n}\right), n \geq 0,
\end{array}\right.
$$

where $v$ is a fixed element in $D,\left\{\eta_{n}\right\},\left\{\lambda_{n}\right\},\left\{\gamma_{n}\right\}$ are sequences in $(0,1)$ such that $\lim _{n \rightarrow \infty} \eta_{n}=0$, $\sum_{n=0}^{\infty} \eta_{n}=\infty$ and $\left\{\alpha_{n}\right\}$ is a sequence in $\left[w, \frac{\delta}{k^{2}}\right]$ for some $0<w \leq \frac{\delta}{k^{2}},\left\{a_{n}\right\},\left\{b_{n}\right\},\left\{c_{n}\right\} \in[d, e] \subset(0,1)$ such that $a_{n}+b_{n}+c_{n}=1$. We will prove that $\left\{x_{n}\right\}$ converges convergence strongly to $\tilde{Q}(v)$, where $\tilde{Q}$ is a sunny nonexpansive retracttion of $X$ onto $S(D, B)$ in a uniformly convex and 2-uniformly smooth Banach space.

\section{PRELIMINARIES}

Let $D$ be a nonempty closed convex subset of Banach space $X$ and let $X^{*}$ be the dual space of $X$. We denote the pairing between $X$ and $X^{*}$ by $\langle.,$.$\rangle . For q>1$, the generalized duality mapping $J_{q}: X \rightarrow 2^{X^{*}}$ is defined by

$$
J_{q}=\left\{j \in X^{*}:\langle u, j\rangle=\|u\|^{q},\|j\|=\|u\|^{q-1}\right\}, \quad \forall u \in X .
$$

Recall that $J=J_{2}$ is called the normalized duality mapping as well as $J_{q}(u)=\|u\|^{q-2} J(u)$ for all $u \in X$. Let

$$
S=\{u \in X:\|u\|=1\} .
$$

A Banach space $X$ is said to be uniformly convex if for any $\varepsilon \in(0,2]$ and $u, v \in S$ and $\|u-v\| \geq \varepsilon$ imply there exists $\delta>0$ such that $\left\|\frac{u+v}{2}\right\| \leq 1-\delta$. It is clear that every uniformly convex Banach space is reflexive. A function $\rho:[0, \infty) \rightarrow[0, \infty)$ is said to be the modulus of smoothness of $X$ if

$$
\rho(t)=\sup \left\{\frac{\|u+t v\|+\|u-t v\|}{2}-1:\|u\|=\|v\|=1\right\}, \quad t \geq 0
$$


A Banach spaceb $X$ is said to be uniformly smooth if and only if $\lim _{t \rightarrow 0} \frac{\rho(t)}{t}=0$. For example the $l_{p}$ spaces $(1<p \leq 2)$ are uniformly smooth. Let $q$ be a fixed real number with $1<q \leq 2$. A Banach space $X$ is said to be $q$-uniformly smooth if there exists a constant $a>0$ such that $\rho(t) \leq a t^{q}$ for all $t>0$; see $[3,22]$ and the references therein.

Lemma 2.1. [3] Let $1<q \leq 2$ be real number. A Banach space $X$ is said to be q-uniformly smooth if and only if there exists a constant $k \geq 1$ such that

$$
\frac{1}{2}\left(\|u+v\|^{q}+\|u-v\|^{q}\right) \leq\|u\|^{q}+\|k v\|^{q}, \forall u, v \in X .
$$

The best constant $k$ is called the q-uniformly smoothness constant of $X$.

Lemma 2.2. [22] Let $q$ be a given real number with $1<q \leq 2$ and let $X$ be a $q$-uniformly smooth Banach space. Then

$$
\|u+v\|^{q} \leq\|u\|^{q}+q\left\langle v, J_{q}(u)\right\rangle+2\|k v\|^{q}, \quad \forall u, v \in X,
$$

where $k$ is the q-uniformly smoothness constant of $X$.

Let $D$ be a nonempty closed convex subset of a Banach space $X$. An operator $B: D \rightarrow X$ is said to be accretive if

$$
\langle B u-B v, J(u-v)\rangle \geq 0, \quad \forall u, v \in D .
$$

An operator $B$ of $D$ into $X$ is said to be $\delta$-inverse strongly accretive if there exists a constant $\delta>0$ such that

$$
\langle B u-B v, J(u-v)\rangle \geq \delta\|B u-B v\|^{2}, \quad \forall u, v \in D .
$$

Let $C$ be a subset of $D$ and $Q_{D}$ a mapping of $D$ into $C$. Then $Q_{D}$ is said to be sunny if

$$
Q_{D}\left(Q_{D} u+t\left(u-Q_{D} u\right)\right)=Q_{D} u
$$

whenever $Q_{D} u+t\left(u-Q_{D} u\right) \in D$ for $u \in D$ and $t \geq 0$. A mapping $Q_{D}$ of $D$ into itself is called a retraction if $Q_{D}^{2}=Q_{D}$. A subset $C$ of $D$ is called a sunny nonexpansive retract of $D$ if there exists a sunny nonexpansive retraction from $D$ onto $C$.

Lemma 2.3. [18] Let $D$ be a nonempty closed convex subset of a smooth Banach space $X$ and let $Q_{D}: X \rightarrow D$ be a retraction. Then the following are equivalent:

(a) $Q_{D}$ is sunny and nonexpansive;

(b) $\left\langle u-Q_{D} u, J\left(v-Q_{D} u\right)\right\rangle \leq 0$, for all $u \in X$ and $v \in D$.

(c) $\left\|Q_{D} u-Q_{D} v\right\|^{2} \leq\left\langle u-v, J\left(Q_{D} u-Q_{D} v\right)\right\rangle$, for all $u . v \in X$.

Lemma 2.4. [2] Let $D$ be a nonempty closed convex subset of a smooth Banach space $X$. Let $Q_{D}$ be a sunny nonexpansive retraction from $X$ onto $D$ and let $B$ be an accretive operator of $D$ into $X$. Thus, $S(D, B)=F\left(Q_{D}(I-\alpha B)\right)$ for all $\alpha>0$.

Let $B$ is an $\delta$-inverse strongly accretive operator of $D$ into $X$. If $0<\alpha \leq \frac{\delta}{k^{2}}$ then $I-\alpha B: D \rightarrow X$ is a nonexpansive mapping; see [2] and the references therein.

Lemma 2.5. [21] Let $X$ be Banach space. Then

$$
\|u+v\|^{2} \leq\|v\|^{2}+2\langle u, J(u+v)\rangle, \forall u, v \in X .
$$


Lemma 2.6. [15] Let $X$ be a uniformly convex Banach space. Thus, there exists a continuous strictly increasing convex function $\psi:[0, \infty) \rightarrow[0, \infty)$ such that $\psi(0)=0$ and

$$
\|a x+b z+c \tilde{x}\|^{2} \leq a\|x\|^{2}+b\|z\|^{2}+c\|\tilde{x}\|^{2}-a b \psi(\|x-y\|)
$$

for all $x, z, \tilde{x} \in B_{r}(0)=\{u \in X:\|u\| \leq r\}$ and $a, b, c \in[0,1]$ with $a+b+c=1$.

Lemma 2.7. [14] Let $\left\{x_{n}\right\}$ be sequences of such that there exists a subsequence $\left\{n_{k}\right\}$ of $\{n\}$ such that $x_{n_{k}} \leq x_{n}$ for all $k \in \mathbb{N}$. Then there exists a nondecreasing sequence $\left\{t_{i}\right\} \subset \mathbb{N}$ such that $t_{i} \rightarrow \infty$ and

$$
x_{t_{i}} \leq x_{t_{i}+1} \text { and } x_{i} \leq x_{t_{i}+1}
$$

for all $i \in \mathbb{N}$. In fact $t_{i}=\max \left\{k \leq i: x_{k} \leq x_{k+1}\right\}$.

Lemma 2.8. [4] Let $X$ be a uniformly convex Banach space, $C$ a nonempty closed convex subset of $X$ and $\tilde{T}: C \rightarrow C$ a nonexpansive mapping. If $\left\{w_{k}\right\}$ is a sequence of $C$ such that $w_{k} \rightarrow w_{0}$ and let $\lim _{k \rightarrow \infty}\left\|w_{k}-\tilde{T} w_{k}\right\|=0$, then $w_{0}$ is a fixed point of $\tilde{T}$.

Lemma 2.9. [23] Let $\left\{x_{n}\right\}$ be a sequence of nonnegative real numbers satisfying

$$
x_{n+1} \leq\left(1-\lambda_{n}\right) x_{n}+\gamma_{n}
$$

where $\left\{\lambda_{n}\right\} \subset(0,1)$ and $\left\{\gamma_{n}\right\}$ is a sequence such that $\sum_{n=0}^{\infty} \lambda_{n}=\infty$ and $\limsup _{n \rightarrow \infty} \gamma_{n} \leq 0$ or $\sum_{n=0}^{\infty}\left|\gamma_{n} \lambda_{n}\right|<$ $\infty$. Then $\lim _{n \rightarrow \infty} x_{n}=0$.

\section{Main Results}

Theorem 3.1. Let $D$ be a nonempty, closed and convex subset of a uniformly convex and 2-uniformly smooth Banach space $X$. Let $Q_{D}: X \rightarrow D$ be a sunny nonexpansive retraction and let $B: D \rightarrow X$ be an $\delta$ inverse strongly accretive operator with $S(D, B) \neq \emptyset$. Let $\left\{x_{n}\right\},\left\{z_{n}\right\}$ and $\left\{\tilde{x}_{n}\right\}$ be a sequences generated by

$$
\left\{\begin{array}{l}
x_{1} \in D \\
z_{n}=Q_{D}\left(x_{n}-\alpha_{n} B x_{n}\right), \\
\tilde{x}_{n}=\lambda_{n} x_{n}+\left(1-\lambda_{n}\right) Q_{D}\left[\gamma_{n} z_{n}+\left(1-\gamma_{n}\right) Q_{D}\left(z_{n}-\alpha_{n} B z_{n}\right)\right], \\
x_{n+1}=\eta_{n} v+\left(1-\eta_{n}\right)\left(a_{n} x_{n}+b_{n} z_{n}+c_{n} \tilde{x}_{n}\right), \quad \forall n \geq 1,
\end{array}\right.
$$

where $v$ is a fixed element in $D,\left\{\eta_{n}\right\},\left\{\lambda_{n}\right\},\left\{\gamma_{n}\right\}$ are sequences in $(0,1)$ such that $\lim _{n \rightarrow \infty} \eta_{n}=0$, $\sum_{n=0}^{\infty} \eta_{n}=\infty$ and $\left\{\alpha_{n}\right\}$ is a sequence in $\left[w, \frac{\delta}{k^{2}}\right]$ for some $0<w \leq \frac{\delta}{k^{2}},\left\{a_{n}\right\},\left\{b_{n}\right\},\left\{c_{n}\right\} \in[d, e] \subset(0,1)$ such that $a_{n}+b_{n}+c_{n}=1$. Then $\left\{x_{n}\right\}$ converges strongly to $\tilde{Q}(v)$, where $\tilde{Q}$ is a sunny nonexpansive retracttion of $X$ onto $S(D, B)$.

Proof. Let $q \in S(D, B)$. From Lemma 2.4, we have

$$
q=Q_{D}\left(q-\alpha_{n} B q\right)
$$

Put

$$
y_{n}=Q_{D}\left(z_{n}-\alpha_{n} B z_{n}\right), w_{n}=Q_{D}\left[\gamma_{n} z_{n}+\left(1-\gamma_{n}\right) Q_{D}\left(z_{n}-\alpha_{n} B z_{n}\right)\right] .
$$


Since $I-\alpha_{n} B$ is nonexpancive, we find from (3.1) that

$$
\begin{aligned}
\left\|z_{n}-q\right\| & =\left\|Q_{D}\left(x_{n}-\alpha_{n} B x_{n}\right)-Q_{D}\left(q-\alpha_{n} B q\right)\right\| \\
& \leq\left\|\left(x_{n}-\alpha_{n} B x_{n}\right)-\left(q-\alpha_{n} B q\right)\right\| \\
& \leq\left\|x_{n}-q\right\| .
\end{aligned}
$$

From $\alpha_{n} \in\left[w, \frac{\delta}{k^{2}}\right]$ and Lemma 2.2, we obtain that

$$
\begin{aligned}
\left\|y_{n}-q\right\|^{2} & =\left\|Q_{D}\left(z_{n}-\alpha_{n} B z_{n}\right)-Q_{D}\left(q-\alpha_{n} B q\right)\right\|^{2} \\
& \leq\left\|\left(z_{n}-q\right)-\alpha_{n}\left(B z_{n}-B q\right)\right\|^{2} \\
& \leq\left\|z_{n}-q\right\|^{2}-2 \alpha_{n}\left\langle B z_{n}-B q, J\left(z_{n}-q\right)\right\rangle+2 k^{2} \alpha_{n}^{2}\left\|B z_{n}-B q\right\|^{2} \\
& \leq\left\|z_{n}-q\right\|^{2}-2 \alpha_{n} \delta\left\|B z_{n}-B q\right\|^{2}+2 k^{2} \alpha_{n}^{2}\left\|B z_{n}-B q\right\|^{2} \\
& =\left\|z_{n}-q\right\|^{2}+2 \alpha_{n}\left(\alpha_{n} k^{2}-\delta\right)\left\|B z_{n}-B q\right\|^{2} \\
& \leq\left\|z_{n}-q\right\|^{2} .
\end{aligned}
$$

From (3.2) and (3.3), we have that

$$
\left\|y_{n}-q\right\| \leq\left\|x_{n}-q\right\|
$$

From $q=Q_{D}(q)$, we get

$$
q=Q_{D}\left[\gamma_{n} q+\left(1-\gamma_{n}\right) Q_{D}\left(q-\alpha_{n} B q\right)\right]
$$

It follows from (3.2) that

$$
\begin{aligned}
\left\|w_{n}-q\right\| & =\left\|Q_{D}\left[\gamma_{n} z_{n}+\left(1-\gamma_{n}\right) Q_{D}\left(z_{n}-\alpha_{n} B z_{n}\right)\right]-Q_{D}\left[\gamma_{n} q+\left(1-\gamma_{n}\right) Q_{D}\left(q-\alpha_{n} B q\right)\right]\right\| \\
& \leq\left\|\left[\gamma_{n} z_{n}+\left(1-\gamma_{n}\right) Q_{D}\left(z_{n}-\alpha_{n} B z_{n}\right)\right]-\left[\gamma_{n} q+\left(1-\gamma_{n}\right) Q_{D}\left(q-\alpha_{n} B q\right)\right]\right\| \\
& \leq \gamma_{n}\left\|z_{n}-q\right\|+\left(1-\gamma_{n}\right)\left\|\left(z_{n}-\alpha_{n} B z_{n}\right)-\left(q-\alpha_{n} B q\right)\right\| \\
& \leq \gamma_{n}\left\|x_{n}-q\right\|+\left(1-\gamma_{n}\right)\left\|x_{n}-q\right\| \\
& =\left\|x_{n}-q\right\| .
\end{aligned}
$$

This implies that

$$
\begin{aligned}
\left\|\tilde{x}_{n}-q\right\| & =\left\|\lambda_{n}\left(x_{n}-q\right)+\left(1-\lambda_{n}\right)\left(w_{n}-q\right)\right\| \\
& \leq \lambda_{n}\left\|x_{n}-q\right\|+\left(1-\lambda_{n}\right)\left\|w_{n}-q\right\| \\
& \leq \lambda_{n}\left\|x_{n}-q\right\|+\left(1-\lambda_{n}\right)\left\|x_{n}-q\right\| \\
& =\left\|x_{n}-q\right\| .
\end{aligned}
$$

We have from (3.1), (3.2) and (3.4) that

$$
\begin{aligned}
\left\|x_{n+1}-q\right\| & =\left\|\eta_{n}(v-q)+\left(1-\eta_{n}\right)\left(a_{n} x_{n}+b_{n} z_{n}+c_{n} \tilde{x}_{n}-q\right)\right\| \\
& \leq \eta_{n}\|v-q\|+\left(1-\eta_{n}\right)\left\|a_{n} x_{n}+b_{n} z_{n}+c_{n} \tilde{x}_{n}-q\right\| \\
& \leq \eta_{n}\|v-q\|+\left(1-\eta_{n}\right)\left(a_{n}\left\|x_{n}-q\right\|+b_{n}\left\|z_{n}-q\right\|+c_{n}\left\|\tilde{x}_{n}-q\right\|\right) \\
& \leq \eta_{n}\|v-q\|+\left(1-\eta_{n}\right)\left(a_{n}\left\|x_{n}-q\right\|+b_{n}\left\|x_{n}-q\right\|+c_{n}\left\|x_{n}-q\right\|\right) \\
& \leq \eta_{n}\left\|v_{n}-q\right\|+\left(1-\eta_{n}\right)\left\|x_{n}-q\right\| \\
& \leq \max \left\{\|v-q\|,\left\|x_{0}-q\right\|\right\} .
\end{aligned}
$$


Thus, $\left\{x_{n}\right\}$ is bounded, and so are $\left\{z_{n}\right\},\left\{\tilde{x}_{n}\right\},\left\{B x_{n}\right\},\left\{B z_{n}\right\},\left\{y_{n}\right\},\left\{w_{n}\right\}$. Let $\tilde{Q}$ be a sunny nonexpansive retraction of $X$ onto $S(D, B)$. Observe that

$$
\begin{aligned}
\left\|z_{n}-\tilde{Q} v\right\| & =\left\|Q_{D}\left(x_{n}-\alpha_{n} B x_{n}\right)-Q_{D}(\tilde{Q} v)\right\| \\
& \leq\left\|\left(x_{n}-\alpha_{n} B x_{n}\right)-\tilde{Q} v\right\| \\
& \leq\left\|x_{n}-\tilde{Q} v\right\| .
\end{aligned}
$$

It follows that

$$
\begin{aligned}
\left\|y_{n}-\tilde{Q} v\right\| & =\left\|Q_{D}\left(z_{n}-\alpha_{n} B z_{n}\right)-Q_{D}(\tilde{Q} v)\right\| \\
& \leq\left\|\left(z_{n}-\alpha_{n} B z_{n}\right)-\tilde{Q} v\right\| \\
& \leq\left\|z_{n}-\tilde{Q} v\right\| \\
& \leq\left\|x_{n}-\tilde{Q} v\right\| .
\end{aligned}
$$

From (3.1) and (3.6) we have

$$
\begin{aligned}
\left\|w_{n}-\tilde{Q} v\right\| & =\left\|Q_{D}\left(\gamma_{n} z_{n}+\left(1-\gamma_{n}\right) Q_{D}\left(z_{n}-\alpha_{n} B z_{n}\right)\right)-Q_{D}(\tilde{Q} v)\right\| \\
& \leq\left\|\gamma_{n}\left(z_{n}-\tilde{Q} v\right)+\left(1-\gamma_{n}\right)\left(Q_{D}\left(z_{n}-\alpha_{n} B z_{n}\right)-\tilde{Q} v\right)\right\| \\
& \leq \gamma_{n}\left\|z_{n}-\tilde{Q} v\right\|+\left(1-\gamma_{n}\right)\left\|Q_{D}\left(z_{n}-\alpha_{n} B z_{n}\right)-\tilde{Q} v\right\| \\
& \leq\left\|x_{n}-\tilde{Q} v\right\|
\end{aligned}
$$

which implies that

$$
\begin{aligned}
\left\|\tilde{x}_{n}-\tilde{Q} v\right\| & =\left\|\lambda_{n}\left(x_{n}-\tilde{Q} v\right)+\left(1-\lambda_{n}\right)\left(w_{n}-\tilde{Q} v\right)\right\| \\
& \leq \lambda_{n}\left\|x_{n}-\tilde{Q} v\right\|+\left(1-\lambda_{n}\right)\left\|w_{n}-\tilde{Q} v\right\| \\
& \leq \lambda_{n}\left\|x_{n}-\tilde{Q} v\right\|+\left(1-\lambda_{n}\right)\left\|x_{n}-\tilde{Q} v\right\| \\
& =\left\|x_{n}-\tilde{Q} v\right\| .
\end{aligned}
$$

From Lemma 2.5, Lemma 2.6, (3.1), (3.5) and (3.7), we have

$$
\begin{aligned}
\left\|x_{n+1}-\tilde{Q} v\right\|^{2}= & \left\|\eta_{n}(v-\tilde{Q} v)+\left(1-\eta_{n}\right)\left(a_{n} x_{n}+b_{n} z_{n}+c_{n} \tilde{x}_{n}-\tilde{Q} v\right)\right\|^{2} \\
\leq & \left(1-\eta_{n}\right)\left\|\left(a_{n} x_{n}+b_{n} z_{n}+c_{n} \tilde{x}_{n}-\tilde{Q} v\right)\right\|^{2}+2 \eta_{n}\left\langle v-\tilde{Q} v, J\left(x_{n+1}-\tilde{Q} v\right)\right\rangle \\
\leq & \left(1-\eta_{n}\right) a_{n}\left\|x_{n}-\tilde{Q} v\right\|^{2}+\left(1-\eta_{n}\right) b_{n}\left\|z_{n}-\tilde{Q} v\right\|^{2}+\left(1-\eta_{n}\right) c_{n}\|\tilde{x}-\tilde{Q} v\|^{2} \\
& -\left(1-\eta_{n}\right) a_{n} b_{n} \psi\left(\left\|x_{n}-z_{n}\right\|\right)+2 \eta_{n}\left\langle v-\tilde{Q} v, J\left(x_{n+1}-\tilde{Q} v\right)\right\rangle \\
\leq & \left(1-\eta_{n}\right)\left[a_{n}\left\|x_{n}-\tilde{Q} v\right\|^{2}+b_{n}\left\|x_{n}-\tilde{Q} v\right\|^{2}+c_{n}\left\|x_{n}-\tilde{Q} v\right\|^{2}\right. \\
& -\left(1-\eta_{n}\right) a_{n} b_{n} \psi\left(\left\|x_{n}-z_{n}\right\|\right)+2 \eta_{n}\left\langle v-\tilde{Q} v, J\left(x_{n+1}-\tilde{Q} v\right)\right\rangle \\
\leq & \left\|x_{n}-\tilde{Q} v\right\|^{2}-\left(1-\eta_{n}\right) a_{n} b_{n} \psi\left(\left\|x_{n}-z_{n}\right\|\right)+2 \eta_{n}\|v-\tilde{Q} v\|\left\|x_{n+1}-\tilde{Q} v\right\| .
\end{aligned}
$$

Similarly, we have

$$
\left\|x_{n+1}-\tilde{Q} v\right\|^{2} \leq\left\|x_{n}-\tilde{Q} v\right\|^{2}-\left(1-\eta_{n}\right) a_{n} c_{n} \psi\left(\left\|x_{n}-\tilde{x}_{n}\right\|\right)+2 \eta_{n}\|v-\tilde{Q} v\|\left\|x_{n+1}-\tilde{Q} v\right\| .
$$

Next, we split the proof into two cases.

Case 1. Assume that there exists $m_{0} \in \mathbb{N}$ such that $\left\{\left\|x_{n}-\tilde{Q} v\right\|\right\}$ is decreasing for all $n \geq m_{0}$. Therefore, we obtain that $\lim _{n \rightarrow \infty}\left\|x_{n}-\tilde{Q} v\right\|=d$. Since $\lim _{n \rightarrow \infty} \gamma_{n}=0$ and $\lim _{n \rightarrow \infty} \eta_{n}=0$, we conclude from (3.8), (3.9) that

$$
\lim _{n \rightarrow \infty} a_{n} c_{n} \psi\left(\left\|x_{n}-\tilde{x}_{n}\right\|\right)=\lim _{n \rightarrow \infty} a_{n} b_{n} \psi\left(\left\|x_{n}-z_{n}\right\|\right)=0 .
$$


Using the property of $\psi$, we conclude that

$$
\lim _{n \rightarrow \infty}\left\|x_{n}-z_{n}\right\|=\lim _{n \rightarrow \infty}\left\|x_{n}-\tilde{x}_{n}\right\|=0 .
$$

Since

$$
\left\|z_{n}-\tilde{x}_{n}\right\| \leq\left\|z_{n}-x_{n}\right\|+\left\|x_{n}-\tilde{x}_{n}\right\|,
$$

we have $\left\|z_{n}-\tilde{x}_{n}\right\| \rightarrow 0$. Observe that

$$
\begin{aligned}
\left\|z_{n}-y_{n}\right\| & =\left\|Q_{D}\left(x_{n}-\alpha_{n} B x_{n}\right)-Q_{D}\left(z_{n}-\alpha_{n} B z_{n}\right)\right\| \\
& \leq\left\|\left(x_{n}-\alpha_{n} B x_{n}\right)-\left(z_{n}-\alpha_{n} B z_{n}\right)\right\| \\
& \leq\left\|x_{n}-z_{n}\right\| .
\end{aligned}
$$

From (3.10), we have $\lim _{n \rightarrow \infty}\left\|z_{n}-y_{n}\right\|=0$. It follows that

$$
\begin{aligned}
\left\|y_{n}-w_{n}\right\| & =\left\|Q_{D}\left(Q_{D}\left(z_{n}-\alpha_{n} B z_{n}\right)\right)-Q_{D}\left(\gamma_{n} z_{n}+\left(1-\gamma_{n}\right) Q_{D}\left(z_{n}-\alpha_{n} B z_{n}\right)\right)\right\| \\
& \leq\left\|\left(Q_{D}\left(z_{n}-\alpha_{n} B z_{n}\right)\right)-\left(\gamma_{n} z_{n}+\left(1-\gamma_{n}\right) Q_{D}\left(z_{n}-\alpha_{n} B z_{n}\right)\right)\right\| \\
& \leq \gamma_{n}\left\|z_{n}-Q_{D}\left(z_{n}-\alpha_{n} B z_{n}\right)\right\| \\
& \leq\left\|z_{n}-y_{n}\right\| \rightarrow 0
\end{aligned}
$$

as $n \rightarrow \infty$. Since

$$
\left\|z_{n}-w_{n}\right\| \leq\left\|z_{n}-y_{n}\right\|+\left\|y_{n}-w_{n}\right\|,
$$

we have $\left\|z_{n}-w_{n}\right\| \rightarrow 0 n \rightarrow \infty$. Since

$$
\left\|x_{n}-y_{n}\right\| \leq\left\|z_{n}-y_{n}\right\|+\left\|x_{n}-z_{n}\right\|,
$$

we have $\left\|x_{n}-y_{n}\right\| \rightarrow 0$ as $n \rightarrow \infty$. From (3.1) and (3.10), we have

$$
\lim _{n \rightarrow \infty}\left\|x_{n+1}-x_{n}\right\|=\lim _{n \rightarrow \infty}\left\|\eta_{n}\left(v-x_{n}\right)+\left(1-\eta_{n}\right)\left(b_{n}\left(z_{n}-x_{n}\right)+c_{n}\left(\tilde{x}_{n}-x_{n}\right)\right)\right\|=0 .
$$

Now, we prove that

$$
\limsup _{n \rightarrow \infty}\left\langle v-\tilde{Q} v, J\left(x_{n}-\tilde{Q} v\right)\right\rangle \leq 0 .
$$

Observe that $\left\{x_{n}\right\}$ is bounded and $X$ is uniformly convex. We can select a subsequence $\left\{x_{n_{k}}\right\}$ of $\left\{x_{n}\right\}$ such that converges weakly to $u$ and

$$
\limsup _{n \rightarrow \infty}\left\langle v-\tilde{Q} v, J\left(x_{n}-\tilde{Q} v\right)\right\rangle=\limsup _{n_{k} \rightarrow \infty}\left\langle v-\tilde{Q} v, J\left(x_{n_{k}}-\tilde{Q} v\right)\right\rangle \leq 0 .
$$

We first show $u \in S(D, B)$. Since $\alpha_{n}$ is in $\left[w, \frac{\delta}{k^{2}}\right]$ for some $w>0$, we have that $\left\{\alpha_{n_{k}}\right\}$ is bounded. Thus, there exists a subsequence $\left\{\alpha_{n_{k}}\right\}$ of $\left\{\alpha_{n_{k}}\right\}$ converges to $\alpha_{0} \in\left[w, \frac{\delta}{k^{2}}\right]$. We may assume, without loss of generality, that $\alpha_{n_{k}} \rightarrow \alpha_{0}$. Since $Q_{D}$ is nonexpansive, we have

$$
\begin{aligned}
\left\|Q_{D}\left(x_{n_{k}}-\alpha_{0} B x_{n_{k}}\right)-x_{n_{k}}\right\| & \leq\left\|Q_{D}\left(x_{n_{k}}-\alpha_{0} B x_{n_{k}}\right)-z_{n_{k}}\right\|+\left\|z_{n_{k}}-x_{n_{k}}\right\| \\
& \leq\left\|Q_{D}\left(x_{n_{k}}-\alpha_{0} B x_{n_{k}}\right)-Q_{D}\left(x_{n_{k}}-\alpha_{n_{k}} B x_{n_{k}}\right)\right\|+\left\|z_{n_{k}}-x_{n_{k}}\right\| \\
& \leq\left\|\left(x_{n_{k}}-\alpha_{0} B x_{n_{k}}\right)-\left(x_{n_{k}}-\alpha_{n_{k}} B x_{n_{k}}\right)\right\|+\left\|z_{n_{k}}-x_{n_{k}}\right\| \\
& \leq\left\|B x_{n_{k}}\right\|\left\|\alpha_{0}-\alpha_{n_{k}}\right\|+\left\|z_{n_{k}}-x_{n_{k}}\right\| .
\end{aligned}
$$

Since $\left\|z_{n}-x_{n}\right\| \rightarrow 0$ as $n \rightarrow \infty$, we have

$$
\lim _{k \rightarrow \infty}\left\|Q_{D}\left(x_{n_{k}}-\alpha_{0} B x_{n_{k}}\right)-x_{n_{k}}\right\|=0 .
$$


Since $Q_{D}\left(I-\alpha_{0} B\right)$ is nonexpansive, we find from Lemma 2.8 that $u \in F\left(Q_{D}\left(I-\alpha_{0} B\right)\right)$. From Lemma 2.4, we also have $u \in S(D, B)$. Using Lemma 2.3 and (3.13) yields that

$$
\begin{aligned}
\limsup _{n \rightarrow \infty}\left\langle v-\tilde{Q} v, J\left(x_{n}-\tilde{Q} v\right)\right\rangle & =\limsup _{k \rightarrow \infty}\left\langle v-\tilde{Q} v, J\left(x_{n_{k}}-\tilde{Q} v\right)\right\rangle \\
& =\limsup _{k \rightarrow \infty}\langle v-\tilde{Q} v, J(u-\tilde{Q} v)\rangle \\
& \leq 0 .
\end{aligned}
$$

Set

$$
t_{n}=\gamma_{n} z_{n}+\left(1-\gamma_{n}\right) Q_{D}\left(z_{n}-\alpha_{n} B z_{n}\right)
$$

for all $n \geq 0$. Since $\left\|x_{n+1}-x_{n}\right\| \rightarrow 0$ as $n \rightarrow \infty$, this along with (3.14) shows that

$$
\limsup _{k \rightarrow \infty}\left\langle v-\tilde{Q} v, J\left(x_{n+1}-\tilde{Q} v\right)\right\rangle \leq 0 .
$$

From (3.1), (3.5), we have that

$$
\begin{aligned}
\left\|x_{n+1}-\tilde{Q} v\right\|^{2}= & \left\langle\eta_{n}(v-\tilde{Q} v)+\left(1-\eta_{n}\right)\left(\left(a_{n} x_{n}+b_{n} z_{n}+c_{n} \tilde{x}\right)-\tilde{Q} v\right), J\left(x_{n+1}-\tilde{Q} v\right)\right\rangle \\
= & \left.\eta_{n}\left\langle v-\tilde{Q} v, J\left(x_{n+1}-\tilde{Q} v\right)\right\rangle+\left(1-\eta_{n}\right)\left\langle\left(a_{n} x_{n}+b_{n} z_{n}+c_{n} \tilde{x}\right)-\tilde{Q} v\right), J\left(x_{n+1}-\tilde{Q} v\right)\right\rangle \\
\leq & \eta_{n}\left\langle v-\tilde{Q} v, J\left(x_{n+1}-\tilde{Q} v\right)\right\rangle+\left(1-\eta_{n}\right)\left(a_{n}\left\|x_{n}-\tilde{Q} v\right\|\left\|x_{n+1}-\tilde{Q} v\right\|\right. \\
& \left.+b_{n}\left\|z_{n}-\tilde{Q} v\right\|\left\|x_{n+1}-\tilde{Q} v\right\|+c_{n}\left\|\tilde{x}_{n}-\tilde{Q} v\right\|\left\|x_{n+1}-\tilde{Q} v\right\|\right) \\
\leq & \eta_{n}\left\langle v-\tilde{Q} v, J\left(x_{n+1}-\tilde{Q} v\right)\right\rangle+\left(1-\eta_{n}\right)\left(\left(\frac{a_{n}}{2}\left(\left\|x_{n}-\tilde{Q} v\right\|^{2}+\left\|x_{n+1}-\tilde{Q} v\right\|^{2}\right)\right.\right. \\
& \left.+\frac{b_{n}}{2}\left(\left\|x_{n}-\tilde{Q} v\right\|^{2}+\left\|x_{n+1}-\tilde{Q} v\right\|^{2}\right)+\frac{c_{n}}{2}\left(\left\|x_{n}-\tilde{Q} v\right\|^{2}+\left\|x_{n+1}-\tilde{Q} v\right\|^{2}\right)\right) \\
\leq & \left(1-\eta_{n}\right)\left\|x_{n}-\tilde{Q} v\right\|^{2}+2 \eta_{n}\left\langle v-\tilde{Q} v, J\left(x_{n+1}-\tilde{Q} v\right)\right\rangle .
\end{aligned}
$$

Since $\lim _{n \rightarrow \infty} \eta_{n}=0, \sum_{n=0}^{\infty} \eta_{n}=\infty$, we obtain from Lemma 2.9, (3.15) and (3.16) that

$$
\lim _{n \rightarrow \infty}\left\|x_{n}-\tilde{Q} v\right\|=0
$$

Case 2. Assume that there exists a subsequence $\left\{n_{k}\right\}$ of $\{n\}$ such that

$$
\left\|x_{n_{k}}-\tilde{Q} v\right\| \leq\left\|x_{n_{k}+1}-\tilde{Q} v\right\|
$$

for all $k \in \mathbb{N}$. From Lemma 2.7, there exists a nondecreasing sequence $\left\{t_{i}\right\} \subset \mathbb{N}$ such that $t_{i} \rightarrow \infty$ and

$$
\left\|x_{t_{i}}-\tilde{Q} v\right\| \leq\left\|x_{t_{i}+1}-\tilde{Q} v\right\| \text { and }\left\|x_{i}-\tilde{Q} v\right\| \leq\left\|x_{t_{i}+1}-\tilde{Q} v\right\|
$$

for all $i \in \mathbb{N}$. Since $\lim _{n \rightarrow \infty} \eta_{n}=0$, we can obtain from (3.8) and (3.9) that

$$
\lim _{i \rightarrow \infty}\left\|x_{t_{i}}-z_{t_{i}}\right\|=\lim _{i \rightarrow \infty}\left\|z_{t_{i}}-y_{t_{i}}\right\|=\lim _{i \rightarrow \infty}\left\|x_{t_{i}}-\tilde{x}_{t_{i}}\right\|=\lim _{i \rightarrow \infty}\left\|x_{t_{i}}-z_{t_{i}}\right\|=\lim _{i \rightarrow \infty}\left\|x_{t_{i}+1}-x_{t_{i}}\right\|=0 .
$$

From Case 1, we also have

$$
\limsup _{i \rightarrow \infty}\left\langle v-\tilde{Q} v, J\left(x_{t_{i}}-\tilde{Q} v\right)\right\rangle \leq 0
$$

and

$$
\limsup _{i \rightarrow \infty}\left\langle v-\tilde{Q} v, J\left(x_{t_{i}+1}-\tilde{Q} v\right)\right\rangle \leq 0
$$

From (3.16), we have

$$
\left\|x_{t_{i}+1}-\tilde{Q} v\right\|^{2} \leq\left(1-\eta_{t_{i}}\right)\left\|x_{t_{i}}-\tilde{Q} v\right\|^{2}+2 \eta_{t_{i}}\left\langle v-\tilde{Q} v, J\left(x_{t_{i}+1}-\tilde{Q} v\right)\right\rangle
$$


This implies that

$$
\eta_{t_{i}}\left\|x_{t_{i}}-\tilde{Q} v\right\|^{2} \leq\left\|x_{t_{i}}-\tilde{Q} v\right\|^{2}-\left\|x_{t_{i}+1}-\tilde{Q} v\right\|^{2}+2 \eta_{t_{i}}\left\langle v-\tilde{Q} v, J\left(x_{t_{i}+1}-\tilde{Q} v\right)\right\rangle .
$$

Since $\eta_{t_{i}}>0$, we get from (3.17) that

$$
\left\|x_{t_{i}}-\tilde{Q} v\right\|^{2} \leq 2\left\langle v-\tilde{Q} v, J\left(t_{t_{i}}-\tilde{Q} v\right)\right\rangle .
$$

Thus, (3.18) implies that $\lim _{i \rightarrow \infty}\left\|x_{t_{i}}-\tilde{Q} v\right\|=0$. From (3.19), we have that $\left\|x_{t_{i}+1}-\tilde{Q} v\right\| \rightarrow 0$ as $i \rightarrow \infty$. Using (3.17), we obtain that $\lim _{i \rightarrow \infty}\left\|x_{i}-\tilde{Q} v\right\|=0$. This completes the proof.

If $v=0$ in Theorem 3.1, then we have the following result.

Corollary 3.1. Let $D$ be a nonempty, closed and convex subset of a uniformly convex and 2-uniformly smooth Banach space $X$. Let $Q_{D}: X \rightarrow D$ be a sunny nonexpansive retraction and let $B: D \rightarrow X$ be an $\delta$ inverse strongly accretive operator with $S(D, B) \neq \emptyset$. Let $\left\{x_{n}\right\},\left\{z_{n}\right\}$ and $\left\{\tilde{x}_{n}\right\}$ be a sequences generated by

$$
\left\{\begin{array}{l}
x_{1} \in D, \\
z_{n}=Q_{D}\left(x_{n}-\alpha_{n} B x_{n}\right) \\
\tilde{x}_{n}=\lambda_{n} x_{n}+\left(1-\lambda_{n}\right) Q_{D}\left[\gamma_{n} z_{n}\left(1-\gamma_{n}\right) Q_{D}\left(z_{n}-\alpha_{n} B z_{n}\right)\right], \\
x_{n+1}=\eta_{n} v+\left(1-\eta_{n}\right)\left(a_{n} x_{n}+b_{n} z_{n}+c_{n} \tilde{x}_{n}\right), \quad \forall n \geq 1,
\end{array}\right.
$$

where $v$ is a fixed element in $D,\left\{\eta_{n}\right\},\left\{\lambda_{n}\right\},\left\{\gamma_{n}\right\}$ are sequences in $(0,1)$ such that $\lim _{n \rightarrow \infty} \eta_{n}=0$, $\sum_{n=0}^{\infty} \eta_{n}=\infty$ and $\alpha_{n}$ is a sequence in $\left[w, \frac{\delta}{k^{2}}\right]$, and $\left\{a_{n}\right\},\left\{b_{n}\right\},\left\{c_{n}\right\} \in[d, e] \subset(0,1)$ such that $a_{n}+b_{n}+c_{n}=$ 1. Then $\left\{x_{n}\right\}$ converges strongly to the minimum norm element in $S(D, B)$.

Proof. Using Theorem 3.1, we obtain that $\left\{x_{n}\right\}$ converges strongly to $\tilde{Q}(0)$, which is the minimum norm element in $S(D, B)$.

\section{Applications}

Using Theorem 3.1, we prove some strong convergence theorems in Banach spaces.

Theorem 4.1. Let $D$ be a nonempty, closed and convex subset of a uniformly convex and 2-uniformly smooth Banach space $X$. Let $B: X \rightarrow X$ be an $\delta$-inverse strongly accretive operator with $B^{-1}(0) \neq \emptyset$. Let $\left\{x_{n}\right\},\left\{z_{n}\right\}$ and $\left\{\tilde{x}_{n}\right\}$ be a sequences generated by

$$
\left\{\begin{array}{l}
x_{1} \in D \\
z_{n}=\left(x_{n}-\alpha_{n} B x_{n}\right) \\
\tilde{x}_{n}=\lambda_{n} x_{n}+\left(1-\lambda_{n}\right)\left(\gamma_{n} z_{n}+\left(1-\gamma_{n}\right)\left(z_{n}-\alpha_{n} B z_{n}\right)\right), \\
x_{n+1}=\eta_{n} v+\left(1-\eta_{n}\right)\left(a_{n} x_{n}+b_{n} z_{n}+c_{n} \tilde{x}_{n}\right), n \geq 1,
\end{array}\right.
$$

where $v$ is a fixed element in $D,\left\{\eta_{n}\right\},\left\{\lambda_{n}\right\},\left\{\gamma_{n}\right\}$ are sequences in $(0,1)$ such that $\lim _{n \rightarrow \infty} \eta_{n}=0$, $\sum_{n=0}^{\infty} \eta_{n}=\infty$ and $\alpha_{n}$ is a sequence in $\left[w, \frac{\delta}{k^{2}}\right]$ for some $0<w \leq \frac{\delta}{k^{2}},\left\{a_{n}\right\},\left\{b_{n}\right\},\left\{c_{n}\right\} \in[d, e] \subset(0,1)$ such that $a_{n}+b_{n}+c_{n}=1$. Then $\left\{x_{n}\right\}$ converges strongly to $\tilde{Q}(v)$, where $\tilde{Q}$ is a sunny nonexpansive retracttion of $X$ onto $B^{-1}(0)$.

Proof. Note that $B^{-1}(0)=S(X, B)$ and $Q_{D}=I$, where $I$ is the identity mapping. Using Theorem 3.1, we get the desired result immediately. 
Let $0 \leq k<1$. A mapping $T: D \rightarrow D$ is said to be $k$-strictly pseudocontractive if there exists $j(u-v) \in$ $J(u-v)$ such that

$$
\langle T u-T v, j(u-v)\rangle \leq\|u-v\|^{2}-\frac{1-k}{2}\|(I-T) u-(I-T) v\|^{2}
$$

for all $u, v \in D$. The above inequality can be rewritten as follows:

$$
\langle(I-T) u-(I-T) v, j(u-v)\rangle \geq \frac{1-k}{2}\|(I-T) u-(I-T) v\|^{2} .
$$

Theorem 4.2. Let $D$ be a nonempty, closed and convex subset of a uniformly convex and 2-uniformly smooth Banach space $X$. Let $Q_{D}: X \rightarrow D$ be a sunny nonexpansive retraction and let $T: D \rightarrow D$ be a $k$-strictly pseudocontractive mapping with $F(T) \neq \emptyset$. Let $\left\{x_{n}\right\},\left\{z_{n}\right\}$ and $\left\{\tilde{x}_{n}\right\}$ be a sequences generated by

$$
\left\{\begin{array}{l}
x_{1} \in D \\
z_{n}=Q_{D}\left(x_{n}-\alpha_{n}\left(x_{n}-T x_{n}\right)\right), \\
\tilde{x}_{n}=\lambda_{n} x_{n}+\left(1-\lambda_{n}\right) Q_{D}\left[\gamma_{n} z_{n}+\left(1-\gamma_{n}\right) Q_{D}\left(z_{n}-\alpha_{n}\left(z_{n}-T z_{n}\right)\right)\right], \\
x_{n+1}=\eta_{n} v+\left(1-\eta_{n}\right)\left(a_{n} x_{n}+b_{n} z_{n}+c_{n} \tilde{x}_{n}\right), n \geq 1,
\end{array}\right.
$$

where $v$ is a fixed element in $D,\left\{\eta_{n}\right\},\left\{\lambda_{n}\right\},\left\{\gamma_{n}\right\}$ are sequences in $(0,1)$ such that $\lim _{n \rightarrow \infty} \eta_{n}=0$, $\sum_{n=0}^{\infty} \eta_{n}=\infty$ and $\alpha_{n}$ is a sequence in $\left[w, \frac{\delta}{k^{2}}\right]$ for some $0<w \leq \frac{\delta}{k^{2}},\left\{a_{n}\right\},\left\{b_{n}\right\},\left\{c_{n}\right\} \in[d, e] \subset(0,1)$ such that $a_{n}+b_{n}+c_{n}=1$. Then $\left\{x_{n}\right\}$ converges strongly to $\tilde{Q}(v)$, where $\tilde{Q}$ is a sunny nonexpansive retracttion of $X$ onto $F(T)$.

Proof. Let $B=I-T$. From (4.1), we obtain that $B$ is $\frac{1-k}{2}$-inverse strongly accretive. It is easy to show that $F(T)=S(D, B)=S(D, I-T)$ (see [2]). By using Theorem 3.1, \{x $\left.x_{n}\right\}$ converges strongly to $\tilde{Q}(v)$, where $\tilde{Q}$ is a sunny nonexpansive retracttion of $X$ onto $F(T)$.

Let $D$ be a subset of a smooth Banach space $X$. Let $\delta>0$. An operator $B: D \rightarrow X$ is said to be $\delta$-strongly accretive if

$$
\langle B u-B v, J(u-v)\rangle \geq \delta\|u-v\|^{2}, \quad \forall u, v \in D .
$$

Theorem 4.3. Let $D$ be a nonempty, closed and convex subset of a uniformly convex and 2-uniformly smooth Banach space $X$. Let $Q_{D}: X \rightarrow D$ be a sunny nonexpansive retraction and let $B: D \rightarrow X$ be an $\delta$-strongly accretive and L-Lipschitz continuous operator with $S(D, B) \neq \emptyset$. Let $\left\{x_{n}\right\},\left\{z_{n}\right\}$ and $\left\{\tilde{x}_{n}\right\}$ be a sequences generated by

$$
\left\{\begin{array}{l}
x_{1} \in D \\
z_{n}=Q_{D}\left(x_{n}-\alpha_{n} B x_{n}\right) \\
\tilde{x}_{n}=\lambda_{n} x_{n}+\left(1-\lambda_{n}\right) Q_{D}\left[\gamma_{n} z_{n}+\left(1-\gamma_{n}\right) Q_{D}\left(z_{n}-\alpha_{n} B z_{n}\right)\right] \\
x_{n+1}=\eta_{n} v+\left(1-\eta_{n}\right)\left(a_{n} x_{n}+b_{n} z_{n}+c_{n} \tilde{x}_{n}\right), n \geq 1
\end{array}\right.
$$

where $v$ is a fixed element in $D,\left\{\eta_{n}\right\},\left\{\lambda_{n}\right\},\left\{\gamma_{n}\right\}$ are sequences in $(0,1)$ such that $\lim _{n \rightarrow \infty} \eta_{n}=0$, $\sum_{n=0}^{\infty} \gamma_{n}=\infty$ and $\alpha_{n}$ is a sequence in $\left[w, \frac{\delta}{k^{2} L^{2}}\right]$ for some $0<w \leq \frac{\delta}{k^{2} L^{2}},\left\{a_{n}\right\},\left\{b_{n}\right\},\left\{c_{n}\right\} \in[d, e] \subset(0,1)$ such that $a_{n}+b_{n}+c_{n}=1$. Then $\left\{x_{n}\right\}$ converges strongly to $\tilde{Q}(v)$, where $\tilde{Q}$ is a sunny nonexpansive retracttion of $X$ onto $S(D, B)$. 
Proof. Since $B: D \rightarrow X$ is $\delta$-strongly accretive and $L$-Lipschitz continuous, we have

$$
\langle B u-B v, J(u-v)\rangle \geq \delta\|u-v\|^{2} \geq \frac{\delta}{L^{2}}\|B u-B v\|^{2}
$$

for $u, v \in D$. Hence, $B$ is $\frac{\delta}{L^{2}}$-inverse strongly accretive. By using Theorem 3.1, $\left\{x_{n}\right\}$ converges strongly to $\tilde{Q}(v)$, where $\tilde{Q}$ is a sunny nonexpansive retracttion of $X$ onto $S(D, B)$.

Example 4.1. Let $X=\mathbb{R}$ with Euclidean norm and usual Euclidean inner product. Let $D:=[-2,1]$ and $Q: \mathbb{R} \rightarrow D$ be defined by

$$
Q_{D} x:=\left\{\begin{array}{cc}
-2, & -\infty<x<-2 \\
x, & -2 \leq x \leq 1 \\
1, & 1 \leq x<\infty
\end{array}\right.
$$

and $B: D \rightarrow \mathbb{R}$ be defined by

$$
B x:=\left\{\begin{array}{lc}
0, & x \in[-2,0], \\
\sin x, & x \in(0,1] .
\end{array}\right.
$$

Clearly, $Q_{D}$ is a sunny nonexpansive retracttion, $S(D, B)=[-2,0]$, and $B$ is a accretive. Next, we prove that $B$ is a $\delta$-inverse strongly accretive for $\delta \leq 1$. If $x, y \in[-2,0]$, then

$$
\langle B x-B y, x-y\rangle=0 \geq\|B x-B y\|^{2}=0 .
$$

If $x, y \in(0,1]$, then there exist $t \in(0,1]$ such that (let $x \leq y)$

$$
\begin{aligned}
\sin y-\sin x=\cos t(y-x) & \Leftrightarrow \sin y-\sin x \leq y-x \\
& \Leftrightarrow 0 \geq \sin x-\sin y \geq x-y \\
& \Leftrightarrow(\sin x-\sin y)(\sin x-\sin y) \leq(x-y)(\sin x-\sin y) \\
& \Leftrightarrow|\sin x-\sin y|^{2} \leq\langle x-y, \sin x-\sin y\rangle
\end{aligned}
$$

If $x \in[-2,0]$ and $y \in(0,1]$, then

$$
\begin{gathered}
\sin y<y \Rightarrow \sin y<y-x \Leftrightarrow \sin ^{2} y<(y-x) \sin y, \\
\langle B x-B y, x-y\rangle=\langle-\sin y, x-y\rangle=\langle\sin y, y-x\rangle \geq|B x-B y|^{2}=|0-\sin y|^{2}=\sin ^{2} y .
\end{gathered}
$$

Hence, $\langle B x-B y, x-y\rangle \geq\|B x-B y\|^{2}$ for all $x \in[-2,1], B$ is a $\delta$-inverse strongly accretive for $\delta \leq 1$.

Example 4.2. Let $X=\mathbb{R}^{2}$ with Euclidean norm and usual Euclidean inner product. Let $D:=[-1,2] \times$ $[-1,2]$ and $Q: \mathbb{R}^{2} \rightarrow D$ be defined by 


$$
Q_{D}(x, y):=\left\{\begin{array}{lc}
(-1,-1), & -\infty<x, y<-1, \\
(x, y), & -1 \leq x, y<2, \\
(2,2), & 2 \leq x, y<\infty, \\
(-1, y), & -\infty<x<-1,-1 \leq y<2, \\
(-1,2), & -\infty<x<-1,2 \leq y<\infty, \\
(x, 2), & -1 \leq x<2,2 \leq y<\infty, \\
(x,-1), & -1 \leq x<2,-\infty<y<-1, \\
(2,-1), & 2 \leq x<\infty,-\infty<y<-1, \\
(2, y), & 2 \leq x<\infty,-1 \leq y<2,
\end{array}\right.
$$

and $B: D \rightarrow \mathbb{R}^{2}$ be defined by

$$
B x:= \begin{cases}(0,0), & x, y \in[-1,1], \\ \left(\frac{x^{2}}{4}, \frac{y^{2}}{4}\right), & \text { otherwise. }\end{cases}
$$

Clearly, $Q_{D}$ is a sunny nonexpansive retracttion and $S(D, B)=[-1,1] \times[-1,1]$, and $B$ is a $\delta$-inverse strongly accretive for $\delta \leq 1$. If $x, y \in[-1,1]$, then

$$
\left\langle B(x, y)-B\left(x^{\prime}, y^{\prime}\right),(x, y)-\left(x^{\prime}, y^{\prime}\right)\right\rangle=0 \geq\left\|B(x, y)-B\left(x^{\prime}, y^{\prime}\right)\right\|^{2}=0 .
$$

Otherwise, since $x+x^{\prime}<4$ for all $x, x^{\prime} \in[-1,2]$, we have

$$
\left|\frac{x^{2}}{4}-\frac{x^{\prime 2}}{4}\right|=\frac{1}{4}\left|\left(x-x^{\prime}\right)\left(x+x^{\prime}\right)\right|<\left|x-x^{\prime}\right|
$$

\section{Acknowledgment}

The authors express their gratitude to the Professor J. J. Nieto and referees for their helpful suggestions which improved final version of this paper.

\section{REFERENCES}

[1] M. A. Alghamdi, N. Shahzad, H. Zegeye, Construction of a common solution of a finite family of variational inequality problems for monotone mappings, J. Nonlinear Sci. Appl. 9 (2016), 1645-1657.

[2] K. Aoyama, H. Iiduka, W. Takahashi, Weak convergence of an iterative sequence for accretive operators in Banach spaces, Fixed Point Theory Appl. 2006 (2006), Article ID 35390.

[3] K. Ball, E. A. Carlen, E. H. Lieb, Sharp uniform convexity and smoothness inequalities for trace norms, Inventiones Math. 115 (1994), 463-482.

[4] F.E. Browder, Semicontractive and semiaccretive nonlinear mappings in Banach spaces, Bull. Amer. Math. Soc. 74 (1968), 660-665.

[5] L.C. Ceng, A. Petrusel, J.C. Yao, Y. Yao, Hybrid viscosity extragradient method for systems of variational inequalities, fixed points of nonexpansive mappings, zero points of accretive operators in Banach spaces, Fixed Point Theory 19 (2018), 487-502.

[6] L.C. Ceng, Approximation of common solutions of a split inclusion problem and a fixed-point problem, J. Appl. Numer. Optim. 1 (2019), 1-12.

[7] S.S. Chang, C.F. Wen, J.C. Yao, Zero point problem of accretive operators in Banach spaces, Bull. Malays. Math. Sci. Soc. 42 (2019), 105-118. 
[8] S.Y. Cho, X. Qin, L. Wang, Strong convergence of a splitting algorithm for treating monotone operators, Fixed Point Theory Appl. 2014 (2014), Article ID 94.

[9] B.A.B. Dehaish, et al., Weak and strong convergence of algorithms for the sum of two accretive operators with applications. J. Nonlinear Convex Anal. 16 (2015), 1321-1336.

[10] H. Iiduka, W. Takahashi, M. Toyoda, Approximation of solutions of variational inequalities for monotone mappings, Panam. Math. J. 14 (2004), 49-61.

[11] H. Iiduka, W. Takahashi, Strong convergence theorems for nonexpansive mappings and inverse-strongly monotone mappings, Nonlinear Anal. 61 (2005), 341-350.

[12] H. Iiduka, W. Takahashi, Weak convergence of a projection algorithm for variational inequalities in a Banach space, J. Math. Anal. Appl. 339 (2008), 668-679.

[13] Y. Liu, A modified hybrid method for solving variational inequality problems in Banach spaces, J. Nonlinear Funct. Anal. 2017 (2017), Article ID 31.

[14] P. E. Mainge, Strong convergence of projected subgradient methods for nonsmooth and nonstrictly convex minimization, Set-Valued Anal. 16 (2008), 899-912.

[15] X. Qin, S.Y. Cho, L. Wang, Strong convergence of an iterative algorithm involving nonlinear mappings of nonexpansive and accretive type, Optimization 67 (2018), 1377-1388.

[16] X. Qin, J.C. Yao, Weak convergence of a Mann-like algorithm for nonexpansive and accretive operators, J. Inequal. Appl. 2016 (2016), Article ID 232.

[17] X. Qin, J.C. Yao, Projection splitting algorithms for nonself operators, J. Nonlinear Convex Anal. 18 (2017), 925-935.

[18] S. Reich, Asymptotic behavior of contractions in Banach spaces, J. Math. Anal. Appl. 44 (1973), 57-70.

[19] S. Reich, S. Sabach, Two strong convergence theorems for Bregman strongly nonexpansive operators in reflexive Banach spaces, Nonlinear Anal. 73 (2010), 122-135.

[20] W. Takahashi, M. Toyoda, Weak convergence theorems for nonexpansive mappings and monotone mappings, J. Optim. Theory Appl. 118 (2003), 417-428.

[21] W. Takahashi, Nonlinear Function Analysis, Fixed Point Theory and Its Applications, Yokohama publishers, Yokohama, (2000).

[22] H. K. Xu, Inequalities in Banach spaces with applications, Nonlinear Anal. 16 (1991), 1127-1138.

[23] Z. Xue, H. Zhou, Y.J. Cho, Iterative solutions of nonlinear equations for m-accretive operators in Banach spaces, J. Nonlineaer Convex Anal. 1 (2000), 313-320.

[24] Y. Yao, M. A. Noor, K. I. Noor, Y. C. Liou, On an iterative algorithm for variational inequalities in Banach spaces, Math. Commun. 16 (2011), 95-104. 